\title{
Unified Approach of Least Squares Adjustment - an Application in 3D Geomatics
}

\author{
Dr. Bashar Alsadik \\ CycloMedia Technology B.V., The Netherlands.
}

\begin{abstract}
An application is presented in this paper to an important technique of least square (LS) adjustment using the unified approach. The unified approach is considering the contribution of weights to unknown parameters in a geomatics model similar to observations. Accordingly, the adjusted parameters are governed by their assigned a priori weights which is normally based on the instrument limitations and manufacturers specifications.

The aim of this paper, is to spot the light on such advanced LS techniques which is suitable nowadays to handle the wealth of observations collected from modern sensors and navigation systems embedded in the modern mapping systems.
\end{abstract}

\section{Keywords: Least Squares - unified approach - photogrammetry - ellipsoid of errors}

\section{Introduction}

The conventional LS adjustment techniques in practice and in literature is depending on categorizing different groups of variables which appear in the mathematical model. The measured variables which are normally have a priori variance - covariance are called observations while the unknown variables that should be determined are called unknown parameters (Mikhail 1976, Ghilani and Wolf 2006).

The rapid development in Geomatics, photogrammetry, computer vision, laser scanning, robotics, and satellite navigation in the last three decades necessitate the development of modified adjustment techniques. These adjustment techniques could handle the integration of different collected data in the mathematical models in a more generic and unified way which is called the unified approach of least square adjustment as mentioned in (Mikhail 1976).

Fig. 1 shows an example of modern mobile mapping systems MMS equipped with a panoramic camera, satellite navigation system GNSS, a laser scanner and ground penetration radar GPR. This means that the system is able to collect different types of observations to determine different unknowns like:

- 3D positions and orientations of the vehicle sensors from the navigation system (GNSS+IMU) with uncertainties.

- Laser point cloud $X Y Z$ of mainly road features with uncertainties.

- Images orientations with uncertainties.

The collected information is aimed to be integrated to deliver highly absolute and relative accuracies of measurements aboveground and underground out of images and point clouds. The processing of these observed redundant data to derive the required quality needs like a unified approach of least squares where the a priori weights is crucial in the adjustment. 


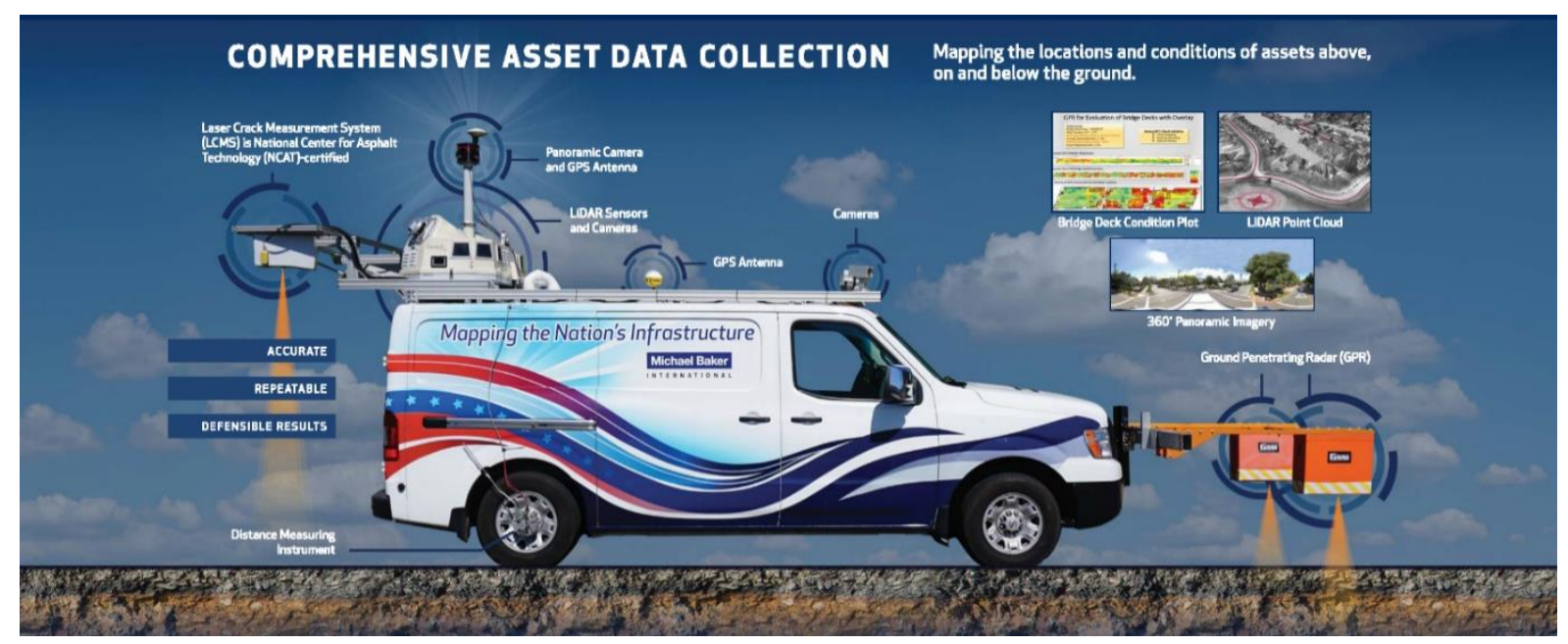

Fig.1.(Baker 2016)

The unified LS adjustment depends on the assumption that all the variables in the mathematical model are observations with a priori variance-covariance matrices (or a priori weights). In another word, it assumes the a priori knowledge of the variables weights in an adjustment problem.

To clarify this weighting problem, we can list two kinds of cases as:

- If an observation is given a very low weight or very a priori high error $(w=0)$, then this means that we can allow this observation to move freely during the adjustment and then it is just like an unknown parameter in the conventional adjustment techniques.

- If there is an observation with a very high weight or very low a priori error, then its weight is infinity as $w \rightarrow \infty$. It means that this observation is not allowed to change during the adjustment and behave like a constant.

Between the above two cases there are a lot of cases in adjustment computations and its worth to mention that in this paper we focus on the nonlinear mathematical problem since it is the case in most of the adjustment problems.

\section{Derivation of the unified LS adjustment}

The derivation is based on the following:

- $\quad l$ as the observations vector, which have a prior cofactor matrix $Q$ and their residuals are $v$.

- The variables that represent unknowns will be treated as observations in the unified approach and therefore will be assigned as vector $x$ with a priori cofactor matrix $Q_{x x}$ and their corrections are $\Delta$.

- There are $n$ total observations in $l$ and $u$ unknowns in $x$ and $\Delta$.

- The redundancy in observations is $r=n-n_{o}$ where $n_{o}$ is the minimum number of observations to solve a problem. 
- The total number of equations is $c=r+u$. In the sense of the unified approach mentioned above, the total observations are $n+u$ and then the redundancy in observations is $\left(n+u-n_{o}\right)=r+u=c$

- Accordingly, the total number of observation equations will be $c+u$ where $c$ is available from the geometric or physical condition of the adjustment problem.

The general observation equations (Ghilani and Wolf 2006) will take the matrix form in equation 1 where the weights $w$ are assigned only to observations.

$A v+B \Delta=F$

Where

$B$ : matrix of partial derivatives in the model to unknowns.

$A$ : matrix of partial derivatives in the model to observations.

$F$ : vector of constants evaluated using the observed values and approximate unknown values.

$\Delta$ : vector of unknown values corrections.

In the sense of the unified LS approach where unknowns are also treated as observations, we add $u$ of new auxiliary observation equations to enable assigning the a priori weights of the unknowns $w_{x x}$. The added auxiliary equations which are linear are modeled by realizing that at the end of the adjustment we get the corrected unknowns $\widehat{\boldsymbol{x}}$ which are similarly treated as adjusted observed variables. In matrix form it means:

$\hat{x}=x^{o}+\Delta=l_{x}+v_{x}$

$v_{x}-\Delta=F_{x}=x^{o}-l_{x}$

Where $x^{o}$ is the vector of approximate values of the unknowns and $l_{x}$ is vector of the observed unknowns themselves and by merging the general observation equation 1 with equation 3 we get:

$\left[\begin{array}{cc}A & 0 \\ 0 & I\end{array}\right]\left[\begin{array}{c}v \\ v_{x}\end{array}\right]+\left[\begin{array}{c}B \\ -I\end{array}\right] \Delta=\left[\begin{array}{c}F \\ F_{x}\end{array}\right]$

Or

$A^{*} v^{*}+B^{*} \Delta^{*}=\dot{F}$

This observation equation system will have the cofactor matrix $\dot{Q}$ of weights as

$\dot{Q}=\left[\begin{array}{cc}Q & 0 \\ 0 & Q_{x x}\end{array}\right] \rightarrow \dot{Q}^{-1}=\dot{w}=\left[\begin{array}{cc}w & 0 \\ 0 & w_{x x}\end{array}\right]$

In (Mikhail 1976), the derivation is continued to observation equation 5 as:

$\dot{Q}_{e}=\dot{A} \dot{Q} \dot{A}^{t}=\left[\begin{array}{cc}A Q A^{t} & 0 \\ 0 & Q_{x x}\end{array}\right]=\left[\begin{array}{cc}Q_{e} & 0 \\ 0 & Q_{x x}\end{array}\right]$
$\dot{N}=\dot{B}^{t} \dot{w} \dot{B}=\left[B^{t} w B+w_{x x}\right]=\left[N+w_{x x}\right]$
$\dot{t}=\dot{B}^{t} \dot{w} \dot{F}=B^{t} w F-w_{x x} F_{x}=\left[t-w_{x x} F_{x}\right]$ 
Accordingly, the normal equations system with the unified approach of least square will be as:

$\left[N+w_{x x}\right] \Delta=\left[t-w_{x x} F_{x}\right]$

The variance-covariance matrix of unknowns after adjustment is computed as:

$Q_{\Delta \Delta}=\left[N+w_{x x}\right]^{-1}$

\section{Application}

To practice the application of the unified approach of least square, two numerical examples in land surveying and photogrammetry are described and solved.

\section{1 application in land surveying}

Given

Four stations $\mathrm{A}, \mathrm{B}, \mathrm{C}$, and $\mathrm{D}$, are intersecting to unknown point $\mathrm{P}$ by four measured distances, four azimuth angles and one difference in height as given below:

$\begin{array}{cccccccc}\text { Station } & \mathrm{X}[\mathrm{m}] & \mathrm{Y}[\mathrm{m}] & \mathrm{Z}[\mathrm{m}] & \text { side } & \text { distances }[\mathrm{m}] & \text { Azimuth }^{\circ} & \text { diff. in Z[m] } \\ \text { A } & 472742.87 & 5191874.15 & 510.56 & \text { AP } & 3.71 & 267.60635^{\circ} & 2.11 \\ \text { B } & 472751.59 & 5191872.49 & 513.76 & \text { BP } & 6.17 & 108.09892^{\circ} & \\ \text { C } & 472738.08 & 5191886.95 & 518.11 & \text { CP } & 15.90 & 328.80011^{\circ} & \\ \text { D } & 472715.23 & 5191852.36 & 515.39 & \text { DP } & 37.82 & 234.94600^{\circ} & \\ \text { P } & 472745.87 & 5191874.29 & 512.68 & & & & \end{array}$

The a priori standard deviations of measurements and unknowns are:

- $\sigma_{d}$ of distances are $\pm 2 \mathrm{~mm}$.

- $\sigma_{A Z}$ of azimuth angles are $\pm 5 "$.

- $\sigma_{X Y Z}$ of $\pm 1 \mathrm{~mm}$ for points $\mathrm{A}, \mathrm{B}, \mathrm{C}$ and D.

- $\sigma_{P}$ of $\pm 10 \mathrm{~mm}$ for point P.

Required:

Apply the unified LS adjustment to find the adjusted positions of the points A, B, C.D and P?

Solution:

The weight $w$ matrices arranged are as follows as mentioned in equation 6:

$$
\begin{aligned}
& \underbrace{w_{D}}_{4 * 4}=\operatorname{diagonal}\left(1 / .002^{2} \ldots \ldots 1 / .002^{2}\right) \\
& \underbrace{w_{A Z}}_{4 * 4}=\operatorname{diagonal}\left(1 / 5^{\prime \prime 2} \ldots \ldots 1 / 5^{\prime \prime 2}\right) \\
& \underbrace{w_{D H}}_{1 * 1}=\left[1 / .005^{2}\right] \\
& \underbrace{w_{X Y Z}}_{12 * 12}=\operatorname{diagonal}\left(1 / .001^{2} \ldots \ldots 1 / .001^{2}\right) \\
& \underbrace{w_{P}}_{3 * 3}=\left[\begin{array}{lll}
1 / .01^{2} & & \\
& 1 / .01^{2} & \\
& & 1 / .01^{2}
\end{array}\right] \\
& W=\operatorname{diagonal}\left(w_{D} \quad w_{A Z} \quad w_{D H} \quad w_{X Y Z} \quad w_{P}\right)
\end{aligned}
$$

It should be noted that we also add 15 linear observation equations ( $3 \times$ number of points $n$ ) in the sense of the unified approach as illustrated for point $A$ : 


$$
\begin{aligned}
& F\left(X_{A}\right)=X_{A}^{o}-X_{A}^{a}=0 \\
& F\left(Y_{A}\right)=Y_{A}^{o}-Y_{A}^{a}=0 \\
& F\left(Z_{A}\right)=Z_{A}^{o}-Z_{A}^{a}=0
\end{aligned}
$$

Where $\boldsymbol{o}$ refers to observed and $\boldsymbol{a}$ for adjusted. The observation equations in matrix form as mentioned in equation 3 can be formed for the points as:

$$
\left[\begin{array}{c}
v_{x A} \\
v_{y A} \\
v_{z A} \\
\vdots \\
v_{x P} \\
v_{y P} \\
v_{z P}
\end{array}\right]=\left[\begin{array}{lllll}
-1 & & & & \\
& -1 & & & \\
& & -1 & & \\
& & & \ddots & \\
& & & & -1
\end{array}\right]\left[\begin{array}{c}
\Delta_{x A} \\
\Delta_{y A} \\
\Delta_{z A} \\
\vdots \\
\Delta_{x P} \\
\Delta_{y P} \\
\Delta_{z P}
\end{array}\right]-\left[\begin{array}{c}
F_{x A} \\
F_{y A} \\
F_{z A} \\
\vdots \\
F_{x P} \\
F_{y P} \\
F_{z P}
\end{array}\right]
$$

\begin{tabular}{|c|c|c|c|c|c|c|c|c|c|c|c|c|c|c|c|c|}
\hline & $d x A$ & dyA & $\mathrm{dzA}$ & $d x B$ & dyB & $d z B$ & $d x C$ & dyc & $\mathrm{dzC}$ & $d x D$ & dyD & $d z D$ & $d x P$ & dyp & $d z P$ & $F$ \\
\hline D-AP & -0.81622 & -0.03915 & -0.57641 & 0 & 0 & 0 & 0 & 0 & 0 & 0 & c & 0 & $0 \quad 0.816221$ & 0.039152 & 0.576412 & 0.076 \\
\hline D-BP & 0 & 0 & 0 & 0.938559 & -0.29611 & 0.177272 & 0 & 0 & 0 & 0 & c & 0 & $\begin{array}{ll}0 & -0.93856\end{array}$ & 0.296111 & -0.17727 & 0.097 \\
\hline D-CP & 0 & 0 & 0 & 0 & 0 & 0 & -0.49244 & 0.799837 & 0.343166 & 0 & c & 0 & $\begin{array}{ll}0 & 0.492441\end{array}$ & -0.79984 & -0.34317 & 0.110 \\
\hline D-DP & 0 & 0 & 0 & 0 & 0 & 0 & 0 & 0 & 0 & -0.81105 & $-0.5805 €$ & 0.07173 & $\begin{array}{ll}3 & 0.81105\end{array}$ & 0.580562 & -0.07173 & 0.076 \\
\hline Az-AP & -0.01065 & 0.221924 & 0 & 0 & 0 & 0 & 0 & 0 & 0 & 0 & c & 0 & $0 \quad 0.010645$ & -0.22192 & 0 & 0.006 \\
\hline Az-BP & 0 & 0 & 0 & -0.0486 & -0.15406 & 0 & 0 & 0 & 0 & 0 & c & 0 & $\begin{array}{ll}0 & 0.048604\end{array}$ & 0.154056 & 0 & 0.010 \\
\hline $\mathrm{Az}-\mathrm{CP}$ & 0 & 0 & 0 & 0 & 0 & 0 & 0.050548 & 0.031121 & 0 & 0 & c & 0 & $\begin{array}{ll}0 & -0.05055\end{array}$ & -0.03112 & 0 & 0.007 \\
\hline Az-DP & 0 & 0 & 0 & 0 & 0 & 0 & 0 & 0 & 0 & -0.01537 & 0.021467 & 0 & $\begin{array}{ll}0 & 0.015367\end{array}$ & -0.02147 & 0 & 0.009 \\
\hline DZ-AP & 0 & 0 & 1 & 0 & 0 & 0 & 0 & 0 & 0 & 0 & c & 0 & 0 & 0 & -1 & .009 \\
\hline
\end{tabular}

For clarity, we list the first two iterations of adjustment as follows:

\begin{tabular}{|c|c|c|c|c|c|c|c|c|c|c|c|c|c|c|c|c|}
\hline & & & & & & & $N-1$ & & & & & & & & T & $\Delta$ \\
\hline $9.31 \mathrm{E}-07$ & $7.59 \mathrm{E}-09$ & $-2.31 E-08$ & $3.17 \mathrm{E}-08$ & $-2.01 \mathrm{E}-08$ & $6.53 \mathrm{E}-09$ & $1.65 E-08$ & 4.32E- -08 & $1.03 E-08$ & 2.03E-08 & $-3.04 \mathrm{E}-08$ & $8.51 E-11$ & $7.02 \mathrm{E}-08$ & $-3.24 \mathrm{E}-08$ & $6.14 \mathrm{E}-07$ & $-9.79 E+04$ & -0.004 \\
\hline $7.59 \mathrm{E}-09$ & $3.93 \mathrm{E}-07$ & $-1.46 E-08$ & $5.42 E-08$ & $19 E-07$ & $-7.95 E-09$ & $3.80 E-08$ & $1.02 E-07$ & $45 E-08$ & $-9.86 \mathrm{E}-08$ & $1.81 \mathrm{E}-07$ & $-1.83 E-09$ & $-1.16 \mathrm{E}-07$ & $3.80 \mathrm{E}-07$ & $-1.39 E-08$ & $1.84 E+06$ & -0.052 \\
\hline$-2.31 \mathrm{E}-08$ & $-1.46 \mathrm{E}-08$ & $9.72 E-07$ & $2.80 \mathrm{E}-08$ & $-1.21 \mathrm{E}-08$ & $5.46 \mathrm{E}-09$ & $-8.15 E-09$ & 4. $25 \mathrm{E}-08$ & $.48 \mathrm{E}-08$ & $2.91 \mathrm{E}-09$ & $-1.57 \mathrm{E}-08$ & 4. $88 \mathrm{E}-10$ & $2.86 \mathrm{E}-08$ & $-1.23 \mathrm{E}-08$ & $6.98 \mathrm{E}-07$ & $-8.90 E+03$ & -0.007 \\
\hline 3.17E-08 & $5.42 E-08$ & $2.80 \mathrm{E}-08$ & $7.98 E-07$ & $-1.33 E-07$ & $-2.75 E-08$ & $1.27 E-07$ & $8.02 E-08$ & $6.21 \mathrm{E}-10$ & 4.16E-08 & $-2.34 \mathrm{E}-09$ & $-2.34 E-09$ & 1.68E-07 & $6.12 \mathrm{E}-08$ & $1.25 \mathrm{E}-07$ & $-8.88 E+05$ & -0.002 \\
\hline$-2.01 E-08$ & $3.19 E-07$ & $-1.21 E-08$ & $-1.33 E-07$ & 4.15E- 07 & $8.86 \mathrm{E}-09$ & $1.72 E-07$ & $1.37 \mathrm{E}-07$ & $9.53 \mathrm{E}-09$ & $-2.04 E-08$ & $1.26 \mathrm{E}-07$ & $-4.09 E-09$ & $9.67 \mathrm{E}-08$ & 3.28E-07 & $-2.22 E-07$ & $-2.90 E+06$ & -0.119 \\
\hline $6.53 E-09$ & $-7.95 E-09$ & $5.46 E-09$ & $-2.75 E-08$ & $8.86 \mathrm{E}-09$ & $9.95 E-07$ & $1.25 \mathrm{E}-08$ & $6.39 \mathrm{E}-09$ & $-4.09 \mathrm{E}-10$ & $8.26 \mathrm{E}-09$ & $-7.23 \mathrm{E}-09$ & $-1.80 \mathrm{E}-10$ & 2.36E-08 & $-7.25 \mathrm{E}-09$ & $3.35 E-08$ & 4.37E+03 & 0.006 \\
\hline $1.65 \mathrm{E}-08$ & $3.80 \mathrm{E}-08$ & $-8.15 E-09$ & $1.27 E-07$ & 1.72E-07 & $1.25 \mathrm{E}-08$ & $6.99 \mathrm{E}-07$ & $-1.60 \mathrm{E}-07$ & $7.80 \mathrm{E}-09$ & 1.53E-07 & $-5.05 E-08$ & $-6.83 E-09$ & 4.96E- 07 & $6.08 \mathrm{E}-08$ & $-5.34 E-07$ & $.95 E+05$ & 0.099 \\
\hline $4.32 E-08$ & $1.02 E-07$ & 4.25E-08 & $8.02 E-08$ & $1.37 \mathrm{E}-07$ & $6.39 \mathrm{E}-09$ & $-1.60 \mathrm{E}-07$ & $7.45 E-07$ & $-4.88 \mathrm{E}-08$ & $3.52 E-08$ & $1.57 \mathrm{E}-08$ & $-2.71 E-09$ & $1.78 \mathrm{E}-07$ & $1.10 \mathrm{E}-07$ & $2.59 \mathrm{E}-07$ & 4.00E+05 & 0.064 \\
\hline $1.03 E-08$ & $2.45 \mathrm{E}-08$ & $1.48 \mathrm{E}-08$ & $6.21 \mathrm{E}-10$ & 9.53E-09 & $-4.09 E-10$ & $7.80 E-09$ & $-4.88 \mathrm{E}-08$ & $9.83 \mathrm{E}-07$ & $-1.83 \mathrm{E}-08$ & $1.45 \mathrm{E}-08$ & 4.63E-10 & $-3.97 E-08$ & $2.24 \mathrm{E}-08$ & $1.83 \mathrm{E}-07$ & $1.07 E+04$ & 0.001 \\
\hline $2.03 \mathrm{E}-08$ & $-9.86 \mathrm{E}-08$ & 2.91E-09 & 4.16E-08 & $-2.04 \mathrm{E}-08$ & $8.26 \mathrm{E}-09$ & $1.53 E-07$ & 3.52E-08 & $-1.83 \mathrm{E}-08$ & 7.83E-07 & $8.48 \mathrm{E}-08$ & 9.17E-09 & $2.58 \mathrm{E}-07$ & $-8.87 E-08$ & $-2.01 E-07$ & $-2.64 E+05$ & -0.093 \\
\hline$-3.04 E-08$ & $1.81 \mathrm{E}-07$ & $-1.57 E-08$ & $-2.34 \mathrm{E}-09$ & $1.26 \mathrm{E}-07$ & $-7.23 \mathrm{E}-09$ & $-5.05 E-08$ & 1.57E- 08 & $1.45 \mathrm{E}-08$ & 8.48E- 08 & 15E-07 & $8.65 E-09$ & $-1.57 \mathrm{E}-07$ & $1.78 \mathrm{E}-07$ & $-2.24 E-08$ & +05 & 0.107 \\
\hline $8.51 E-11$ & $-1.83 \mathrm{E}-09$ & 4.88E-10 & $-2.34 \mathrm{E}-09$ & $-4.09 \mathrm{E}-09$ & $-1.80 \mathrm{E}-10$ & $-6.83 E-09$ & $-2.71 \mathrm{E}-09$ & 4.63E- 10 & $9.17 E-09$ & $8.65 \mathrm{E}-09$ & 9.99E-07 & $-8.52 E-09$ & $-2.25 \mathrm{E}-09$ & $1.27 \mathrm{E}-08$ & $1.50 E+03$ & 0.001 \\
\hline $7.02 E-08$ & $-1.16 \mathrm{E}-07$ & 2.86E-08 & $1.68 \mathrm{E}-07$ & $9.67 E-08$ & $2.36 \mathrm{E}-08$ & 4.96E-07 & $1.78 \mathrm{E}-07$ & $-3.97 E-08$ & $2.58 \mathrm{E}-07$ & $-1.57 \mathrm{E}-07$ & $-8.52 E-09$ & $7.59 E-07$ & $-8.58 \mathrm{E}-08$ & $-3.98 E-07$ & $6.55 E+05$ & 0.066 \\
\hline$-3.24 E-08$ & $3.80 \mathrm{E}-07$ & $-1.23 \mathrm{E}-08$ & $6.12 \mathrm{E}-08$ & $3.28 \mathrm{E}-07$ & $-7.25 \mathrm{E}-09$ & $6.08 E-08$ & $1.10 \mathrm{E}-07$ & $2.24 \mathrm{E}-08$ & $-8.87 \mathrm{E}-08$ & $1.78 \mathrm{E}-07$ & $-2.25 \mathrm{E}-09$ & $-8.58 \mathrm{E}-08$ & $3.82 \mathrm{E}-07$ & $-6.16 E-08$ & $3.31 E+05$ & -0.071 \\
\hline $6.14 \mathrm{E}-07$ & $-1.39 \mathrm{E}-08$ & $6.98 \mathrm{E}-07$ & $1.25 \mathrm{E}-07$ & $-2.22 \mathrm{E}-07$ & $3.35 \mathrm{E}-08$ & $-5.34 E-07$ & $2.59 \mathrm{E}-07$ & $1.83 \mathrm{E}-07$ & $-2.01 E-07$ & $-2.24 \mathrm{E}-08$ & $1.27 E-08$ & $-3.98 \mathrm{E}-07$ & $-6.16 \mathrm{E}-08$ & 7.33E-06 & $-7.65 E+03$ & -0.061 \\
\hline
\end{tabular}

\section{LS adjustment - Iteration 1}

The normal equation matrices $N$ and $t$ are as follows:

\section{LS adjustment - Iteration 2}

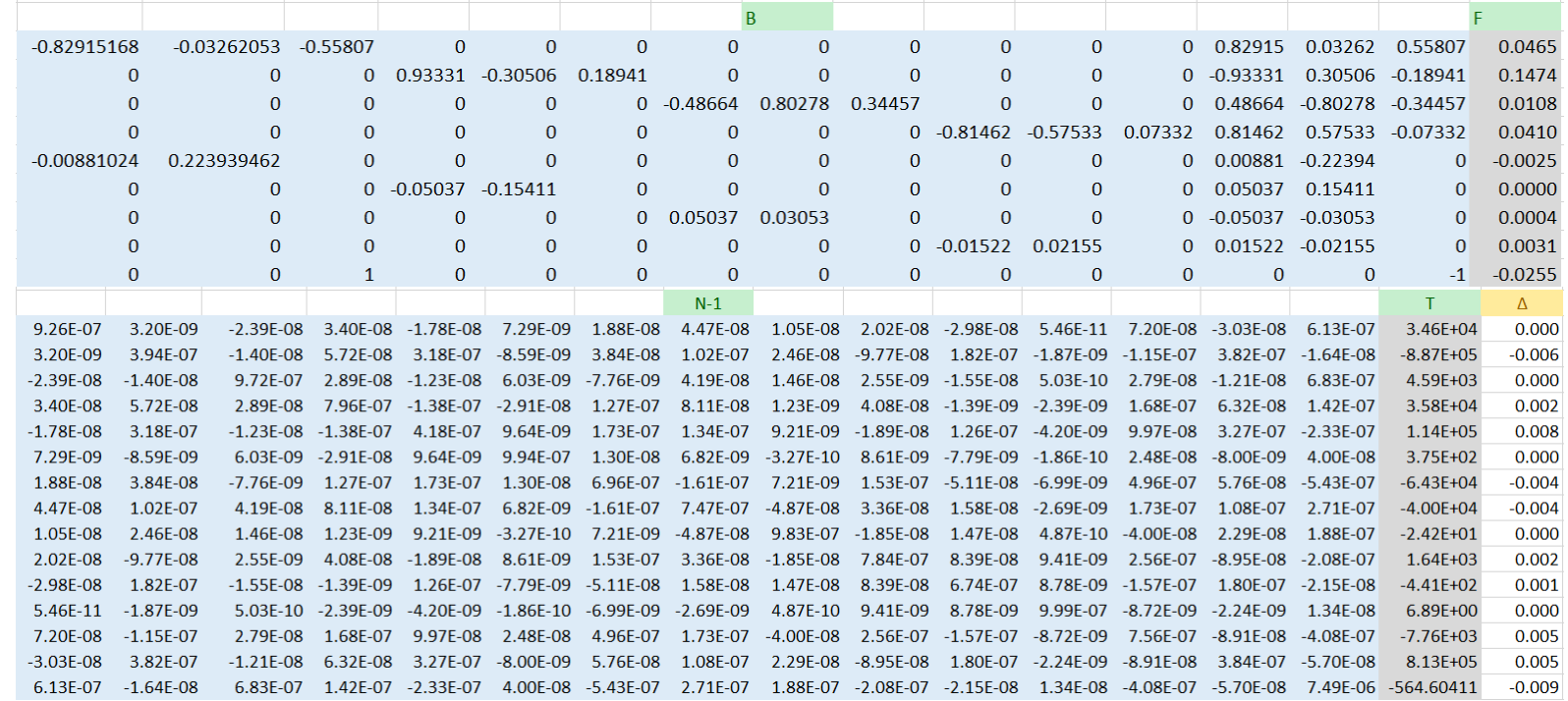


The adjustment iterations continue until reach negligible values (<.0001) as shown in Fig. 2.
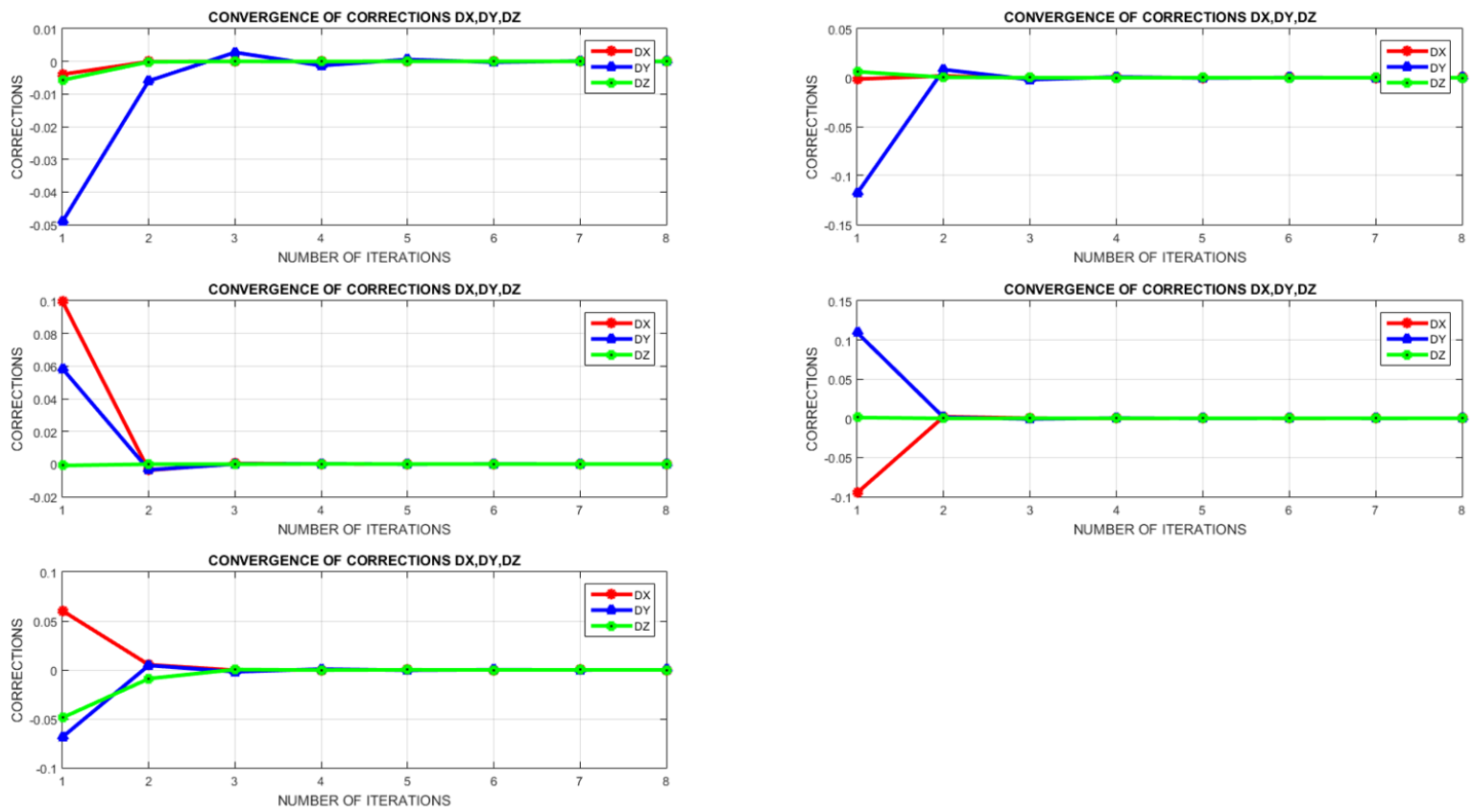

Fig.2. the adjustment iterations of the five points A, B, C, D and P.

The final adjusted points with their coordinates are as follows:

$\begin{array}{ccccccc} & \mathrm{X}[\mathrm{m}] & \mathrm{Y}[\mathrm{m}] & \mathrm{Z}[\mathrm{m}] & \sigma_{X} & \sigma_{Y} & \sigma_{z} \\ \mathrm{~A} & 472,742.87 & 5,191,874.10 & 510.55 & 0.09 & 0.06 & 0.09 \\ \text { B } & 472,751.59 & 5,191,872.38 & 513.77 & 0.08 & 0.06 & 0.09 \\ \text { C } & 472,738.18 & 5,191,887.00 & 518.11 & 0.08 & 0.08 & 0.09 \\ \text { D } & 472,715.14 & 5,191,852.47 & 515.39 & 0.08 & 0.07 & 0.09 \\ \text { P } & 472,745.94 & 5,191,874.22 & 512.62 & 0.08 & 0.06 & 0.25\end{array}$

Fig. 3 illustrates the ellipsoid of errors of the adjusted points.

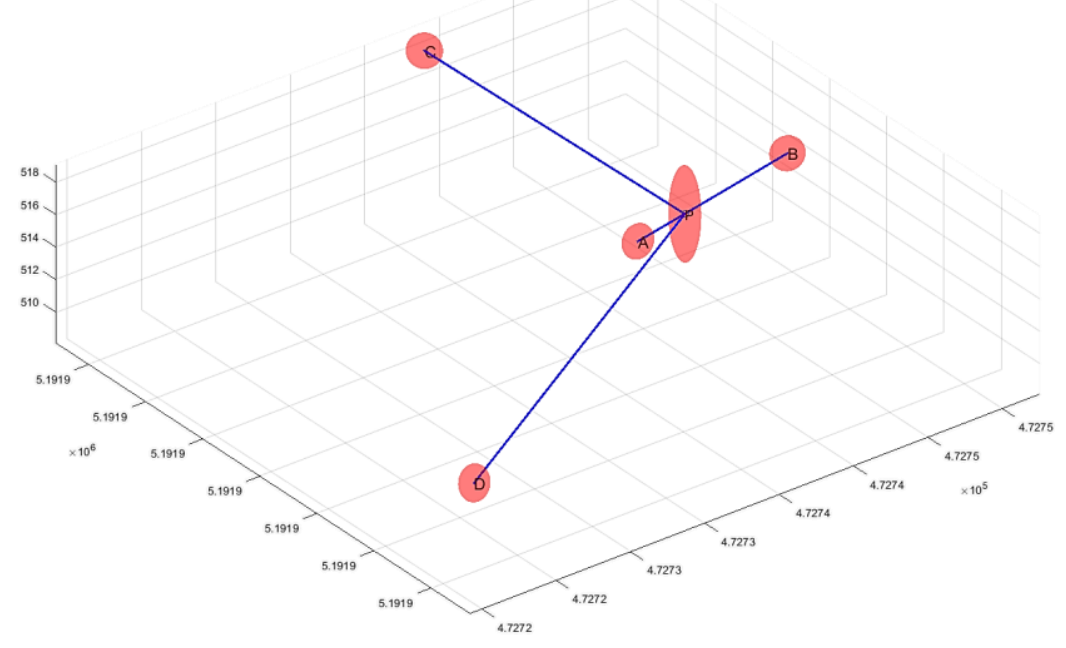

Fig.3. Ellipsoid of errors of the adjusted points with $\sigma_{P}$ of $10 \mathrm{~mm}$. 
To illustrates the effect of weighting on the final adjusted points, we take a high weight of $\mathrm{P}$ as:

- $\sigma_{X Y Z}$ of $\pm 1 \mathrm{~mm}$ for points $\mathrm{A}, \mathrm{B}, \mathrm{C}$ and D.

- $\sigma_{X Y Z}$ of $\pm 1 \mathrm{~mm}$ for point $\mathrm{P}$.

After running the unified LS adjustment with these weights, we get the following results as illustrated in Fig.4:

$\begin{array}{ccccccc} & \mathrm{X}[\mathrm{m}] & \mathrm{Y}[\mathrm{m}] & \mathrm{Z}[\mathrm{m}] & \sigma_{X} & \sigma_{Y} & \sigma_{z} \\ \text { A } & 472,742.87 & 5,191,874.12 & 510.56 & 0.09 & 0.05 & 0.09 \\ \text { B } & 472,751.59 & 5,191,872.39 & 513.77 & 0.08 & 0.05 & 0.09 \\ \text { C } & 472,738.16 & 5,191,887.00 & 518.11 & 0.07 & 0.08 & 0.09 \\ \text { D } & 472,715.13 & 5,191,852.49 & 515.39 & 0.08 & 0.07 & 0.09 \\ \text { P } & 472,745.90 & 5,191,874.24 & 512.68 & 0.06 & 0.05 & 0.09\end{array}$

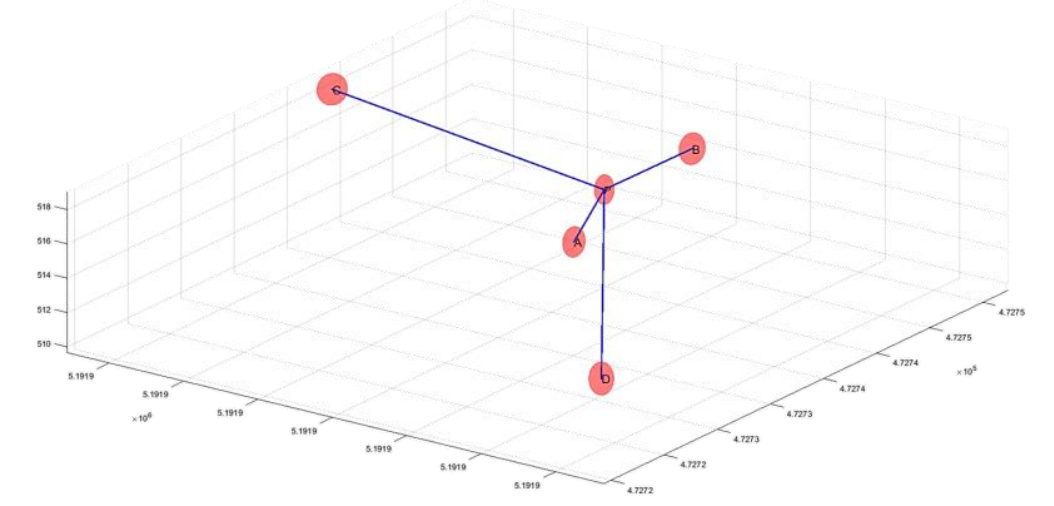

Fig.4. Ellipsoid of errors of the adjusted points with $\sigma_{P}$ of $1 \mathrm{~mm}$.

It is obvious how the high weight assigned to $\mathrm{P}$ is reflected in the unified LS adjustment where the confidence limits are much improved (smaller ellipsoid of errors) in its adjusted position compared to the previous run shown in Fig.3.

\section{2 application in photogrammetry}

A smartphone image is taken with the following settings:

- focal length $=4.15 \mathrm{~mm}$.

- pixel size $=.0015 \mathrm{~mm}$

- image format $=2448 * 3264$ pixels.

- neglected lens distortion.

- Five control points are imaged and their $X Y Z$ coordinates are given with their pixel and transformed coordinates as follows:

$\begin{array}{cccccccc} & X[\mathrm{~m}] & Y[\mathrm{~m}] & Z[\mathrm{~m}] & L n_{\text {pixel }} & S n_{\text {pixel }} & x[\mathrm{~mm}] & y[\mathrm{~mm}] \\ \mathrm{A} & 145899.21 & 424106.18 & 7.15 & 303 & 416 & -0.433 & 0.442 \\ \text { B } & 145904.39 & 424114.19 & 7.16 & 2709 & 490 & -1.398 & 1.845 \\ \text { C } & 145899.71 & 424106.94 & 4.65 & 540 & 1073 & 2.345 & -0.280 \\ \text { D } & 145904.55 & 424114.43 & 5.31 & 2770 & 1816 & 2.253 & 1.732 \\ \text { E } & 145902.38 & 424111.14 & 3.63 & 939 & 1340 & -1.039 & 0.848\end{array}$

- Initial exterior orientation parameters are given as follows where angles are in radian:

$\begin{array}{cccccc}\text { Omega } & \text { phi } & \text { kappa } & \text { Xo[m] } & \text { Yo[m] } & \text { Zo[m] } \\ 1.57 & 1.57 & 0 & 145902.05 & 424110.58 & 5.58\end{array}$


Required:

Apply the unified LS adjustment to find the adjusted image orientation if you consider the following a priori standard deviations of measurements and unknowns:

- $\sigma_{x y}$ of image coordinates are $\pm .003 \mathrm{~mm}$.

- $\sigma_{\text {angle }}$ of orientation angles are $\pm 5^{\prime}$.

- $\sigma_{X o Y o Z o}$ of $\pm 5 \mathrm{~cm}$ for camera coordinates.

- $\quad \sigma_{X Y Z}$ of $\pm 1 \mathrm{~mm}$ for control points $A, B, C, D$ and $E$.

Solution

The observation equations necessary to solve this problem is divided into three types as:

- Collinearity equations $(2 \times$ no. of points $=10)$ with weight $w_{x y}$ (Luhmann T., Robson S. et al. 2006). For point $A$, the collinearity equations is:

$F_{x A}=-f \frac{m_{11}\left(X_{A}-X_{O}\right)+m_{12}\left(Y_{A}-Y_{O}\right)+m_{13}\left(Z_{A}-Z_{o}\right)}{m_{31}\left(X_{p}-X_{o}\right)+m_{32}\left(Y_{A}-Y_{o}\right)+m_{33}\left(Z_{A}-Z_{o}\right)}-x_{A}$
$F_{y A}=-f \frac{m_{21}\left(X_{A}-X_{o}\right)+m_{22}\left(Y_{A}-Y_{O}\right)+m_{23}\left(Z_{A}-Z_{o}\right)}{m_{31}\left(X_{A}-X_{O}\right)+m_{32}\left(Y_{A}-Y_{o}\right)+m_{33}\left(Z_{A}-Z_{o}\right)}-y_{A}$

where

$f$ : focal length in $\mathrm{mm}$.

$m$ 's: rotation matrix elements.

$X_{O}, Y_{O}, Z_{o}$ are the camera coordinates in object coordinate system.

$X_{A}, Y_{A}, Z_{A}$ : coordinates of point $A$ in object system.

$x_{a}, y_{a}$ : observed image coordinates in $\mathrm{mm}$.

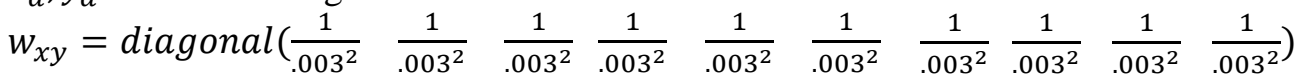

- Six observation equations of the exterior orientation parameters with weight

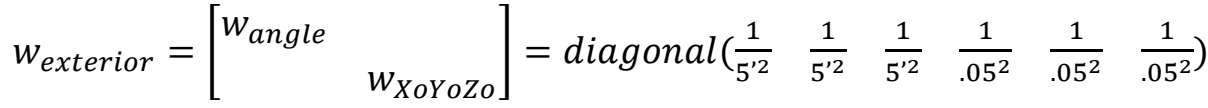

$$
\begin{aligned}
& {\left[\begin{array}{c}
v_{w} \\
v_{\varphi} \\
v_{k} \\
\vdots \\
v_{X o} \\
v_{Y o} \\
v_{Z o}
\end{array}\right]=\left[\begin{array}{lllll}
-1 & & & & \\
& -1 & & & \\
& & -1 & & \\
& & & \ddots & \\
& & & & -1
\end{array}\right]\left[\begin{array}{c}
\Delta_{w} \\
\Delta_{\varphi} \\
\Delta_{k} \\
\vdots \\
\Delta_{X o} \\
\Delta_{Y o} \\
\Delta_{Z o}
\end{array}\right]-\left[\begin{array}{c}
F_{w} \\
F_{\varphi} \\
F_{k} \\
\vdots \\
F_{X o} \\
F_{Y o} \\
F_{Z o}
\end{array}\right]}
\end{aligned}
$$

Like equation 13 , the observation equations of the control points $(3 \times$ no. of points $=15$ equations) with weight $w_{X Y Z}$ are:

$$
\left[\begin{array}{c}
v_{x A} \\
v_{y A} \\
v_{z A} \\
\vdots \\
v_{x E} \\
v_{y} E \\
v_{z E}
\end{array}\right]=\left[\begin{array}{lllll}
-1 & & & & \\
& -1 & & & \\
& & -1 & & \\
& & & \ddots & \\
& & & & -1
\end{array}\right]\left[\begin{array}{c}
\Delta_{x A} \\
\Delta_{y A} \\
\Delta_{z A} \\
\vdots \\
\Delta_{x E} \\
\Delta_{y E} \\
\Delta_{z E}
\end{array}\right]-\left[\begin{array}{c}
F_{x A} \\
F_{y A} \\
F_{z A} \\
\vdots \\
F_{x E} \\
F_{y E} \\
F_{z E}
\end{array}\right]
$$

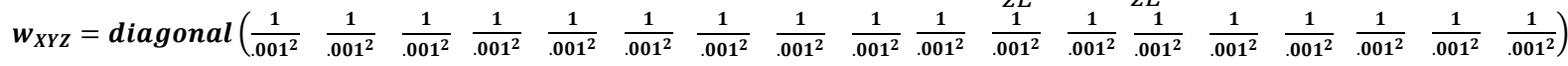
The total weight matrix will be:

$$
w_{31 * 31}=\left[\begin{array}{lll}
w_{x y} & & \\
& w_{\text {exterior }} & \\
& & w_{X Y Z}
\end{array}\right]
$$


LS adjustment - iteration 1

The least square adjustment matrices are:

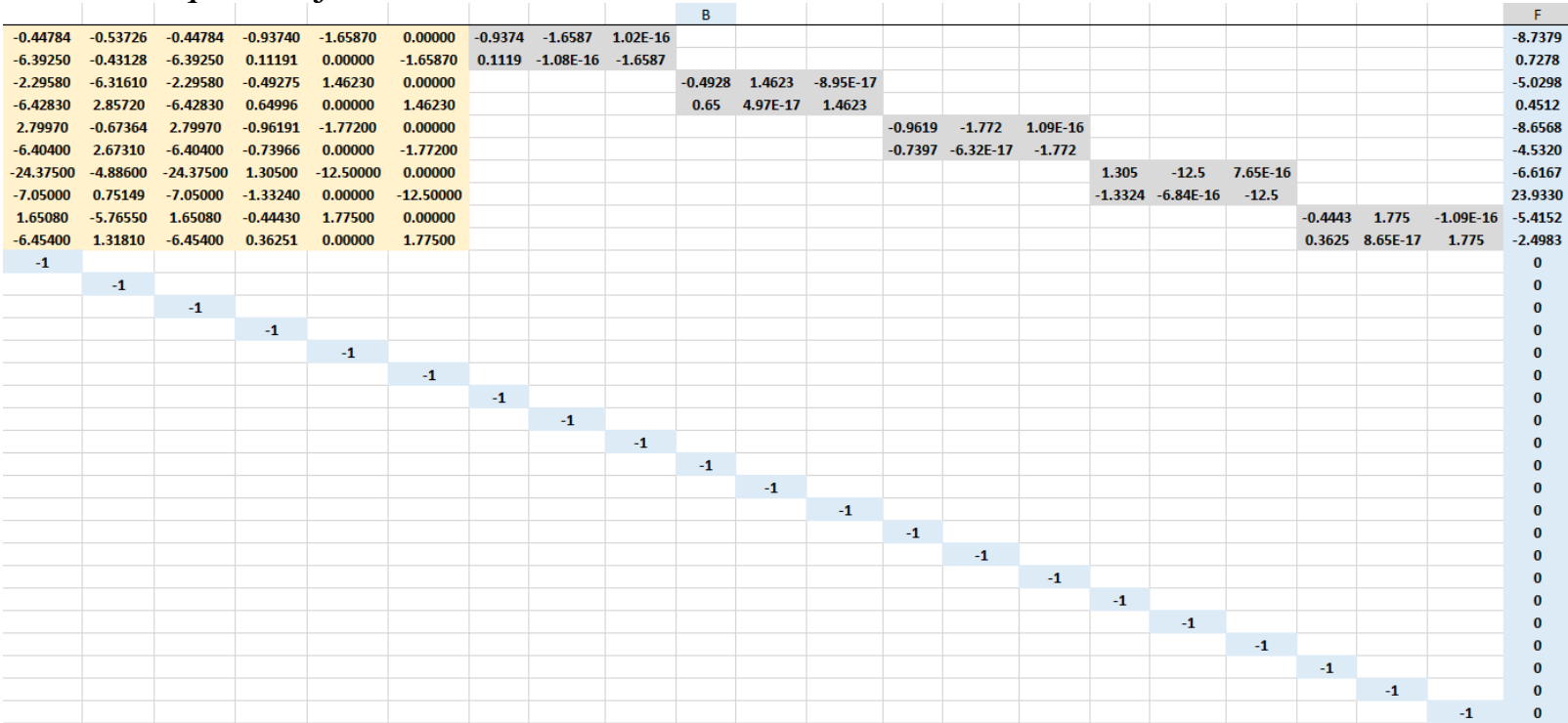

The normal equation matrices are:



LS adjustment - iteration 2

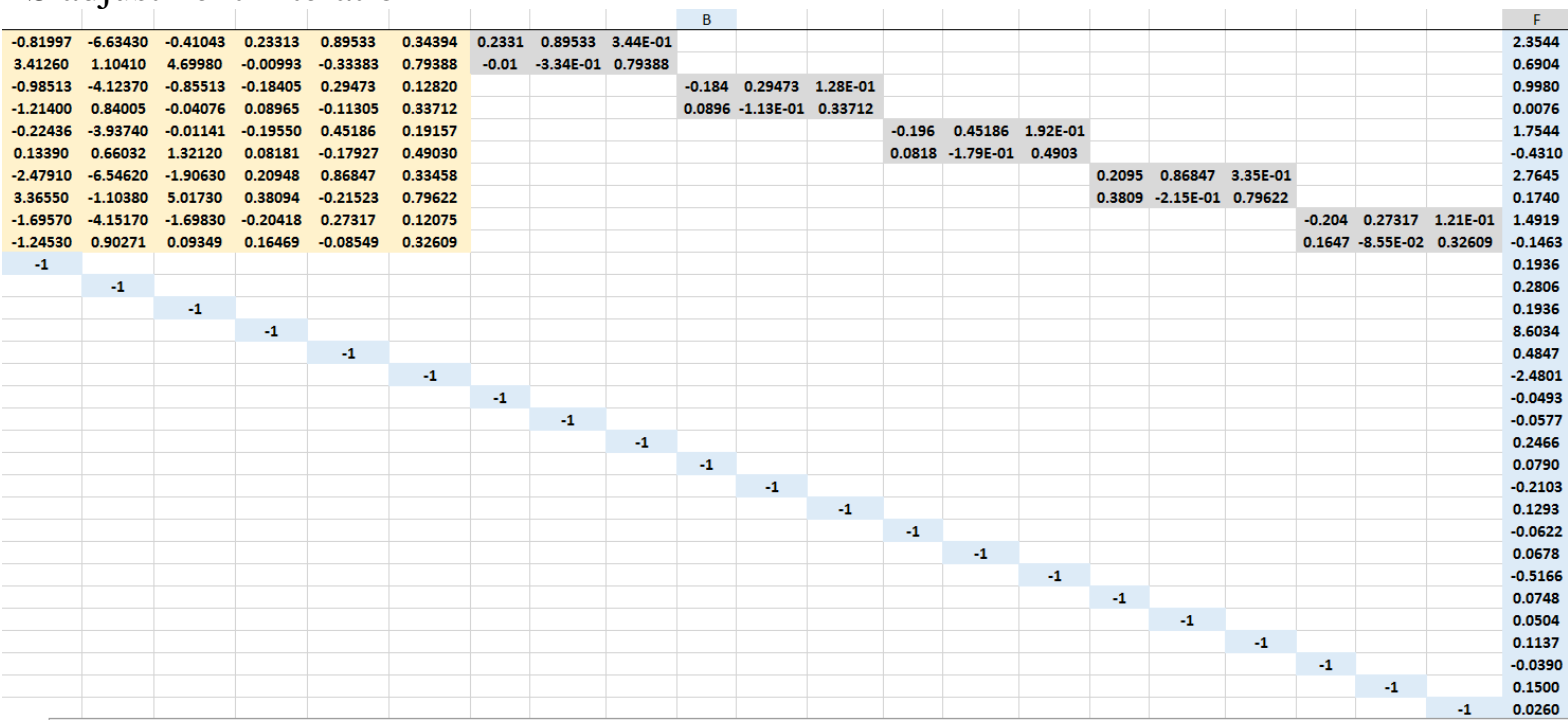

The iterations continue until reach negligible values and becoming stable after five iterations as illustrated in Fig.5 

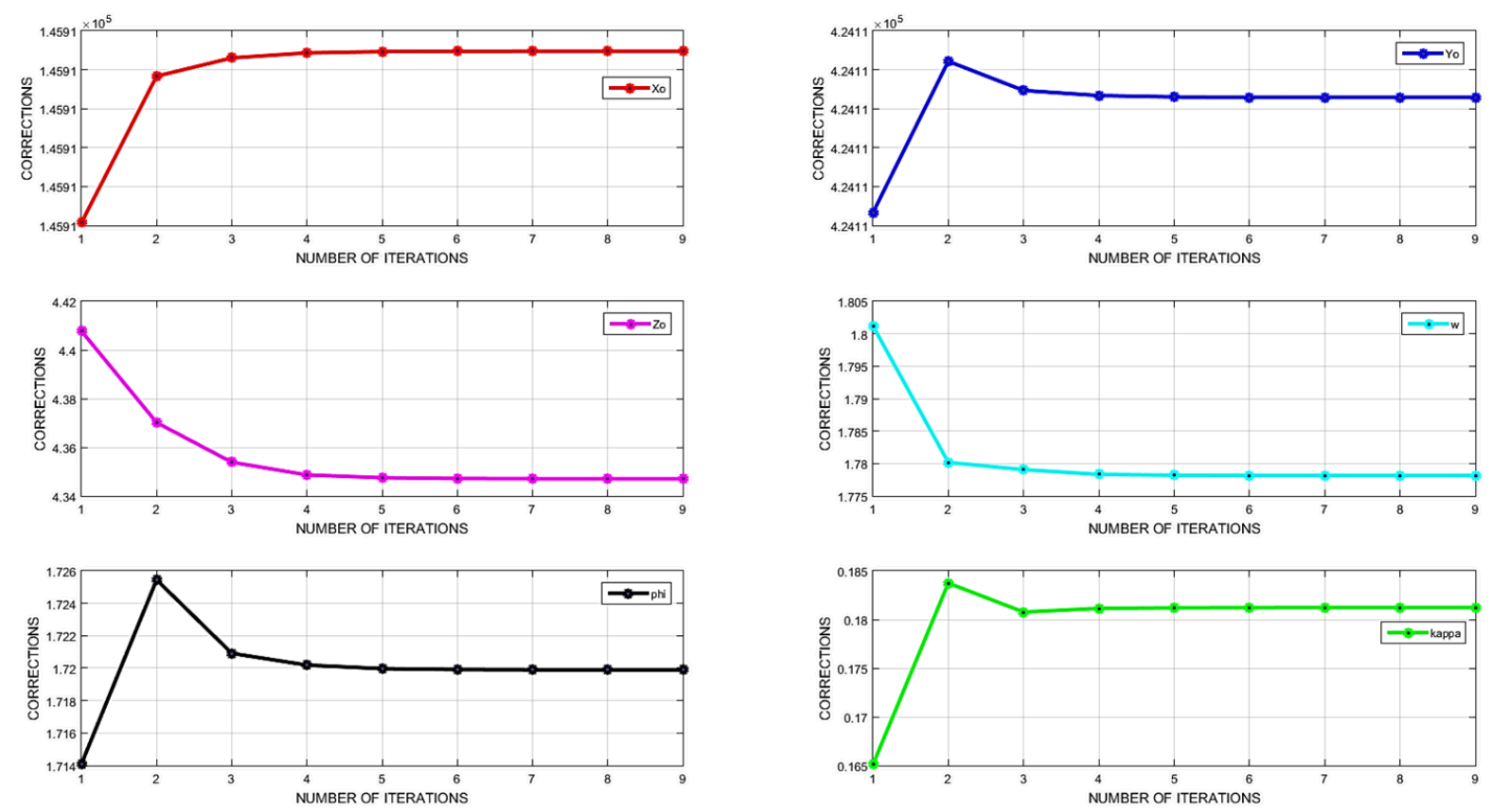

Fig.5. the corrected exterior orientation parameters of the image.

The final adjusted exterior orientation parameters of the image are:

$\begin{array}{cccccc}X o[\mathrm{~m}] & Y o[\mathrm{~m}] & Z o[\mathrm{~m}] & \text { omega[deg.] } & \text { phi }[\mathrm{deg} .] & \text { kappa[deg.] } \\ 145909.624 & 424112.864 & 4.347 & 101.8807 & 98.5428 & 10.3834\end{array}$

To clarify the weight effect on the adjusted camera coordinates and its accuracy, we applied two cases when the a priori standard deviation of control points is $1 \mathrm{~mm}$ and $5 \mathrm{~mm}$ as follows:

\begin{tabular}{ccc|ccc}
\multicolumn{3}{c|}{$\sigma_{X Y Z}=1 \mathrm{~mm}$} & \multicolumn{3}{c}{$\sigma_{X Y Z}=5 \mathrm{~mm}$} \\
$\sigma_{X o}[\mathrm{~m}]$ & $\sigma_{Y o}[\mathrm{~m}]$ & $\sigma_{z o}[\mathrm{~m}]$ & $\sigma_{X o}[\mathrm{~m}]$ & $\sigma_{Y o}[\mathrm{~m}]$ & $\sigma_{z o}[\mathrm{~m}]$ \\
0.078 & 0.081 & 0.041 & 0.128 & 0.115 & 0.063
\end{tabular}
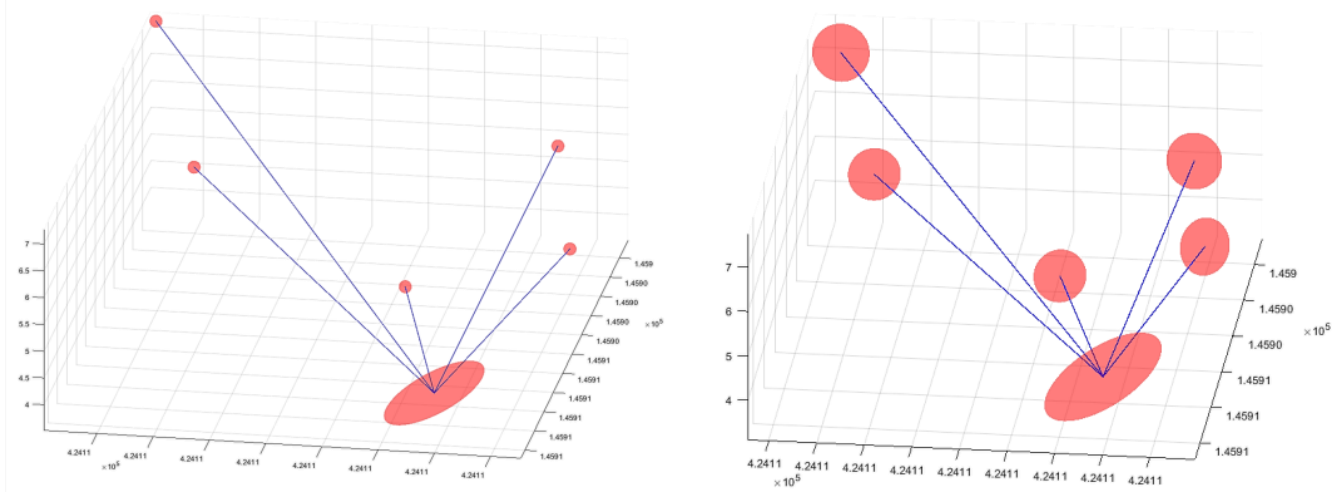

Fig.6. Ellipsoid of errors of the adjusted camera position and the control points.

Left) adjustment with $\sigma_{X Y Z}$ of $1 \mathrm{~mm}$. right) adjustment with $\sigma_{X Y Z}$ of $5 \mathrm{~mm}$.

These two weight settings illustrate the effect of the a priori weights of control points on the adjusted camera coordinates. When using highly trusted control points (smaller $\sigma_{X Y Z}$ ) we get higher trusted camera coordinates (Fig.6a) while using less trusted control points (larger $\sigma_{X Y Z}$ ) results in less trusted camera coordinates (Fig.6b). 


\section{Conclusions}

In this review paper, the unified approach of least square is presented and evaluated through the application of two tests. The two computational tests showed the effectiveness of such adjustment approach to handle the multi-weighted geomatical problems where all the variables in the model are treated as observations. The effect of the different weights on the final adjusted unknowns are also tested and visualized in Figures 4 and 6.

\section{References}

Baker, M. (2016). "Mobile Lidar." 2017, from http://www.mobilelidar.com/2016 0601 archive.html.

Ghilani, C. D. and P. R. Wolf (2006). Adjustment Computations: Spatial Data Analysis, John Wiley \& Sons Inc.

Luhmann T., et al. (2006). Close Range Photogrammetry Principles, Methods and Applications, Whittles Publishing Country, United Kingdom

Mikhail, E. (1976). Observations and Least Squares, University Press of America, Washington, DC. 